http://dx.doi.org/10.35381/r.k.v6i4.1505

\title{
La música como estrategia metodológica para fortalecer el desarrollo integral en los estudiantes
}

\section{Music as a methodological strategy to strengthen the integral development of students}

\author{
Jener Fabricio Chica-Correa \\ jener.chica.60@est.ucacue.edu.ec \\ Universidad Católica de Cuenca, Azogues \\ Ecuador \\ https://orcid.org/0000-0002-3733-1984 \\ María Isabel Álvarez-Lozano \\ mialvarezl@ucacue.edu.ec \\ Universidad Católica de Cuenca, Azogues \\ Ecuador \\ https://orcid.org/0000-0001-8029-1933 \\ Claudio Fernando Guevara-Vizcaíno \\ cfguevarav@ucacue.edu.ec \\ Universidad Católica de Cuenca, Cuenca \\ Ecuador \\ https://orcid.org/0000-0003-3593-0606
}

Recepción: 30 de agosto 2021

Revisado: 20 de septiembre 2021

Aprobación: 15 de noviembre 2021

Publicación: 01 de diciembre 2021 
Revista Arbitrada Interdisciplinaria KOINONIA

Año VI. Vol VI. N4. Edición Especial: Educación III. 2021

Hecho el depósito de Ley: FA2016000010 ISSN: 2542-3088

FUNDACIÓN KOINONIA (F.K). Santa Ana de Coro. Venezuela.

Jener Fabricio Chica-Correa; María Isabel Álvarez-Lozano; Claudio Fernando Guevara-Vizcaíno

\title{
RESUMEN
}

La aplicación de estrategias lúdicas para mejorar los procesos de enseñanza en las instituciones educativas es oportuna, porque transforma la transmisión de conocimientos en procesos interesantes. Por ende, esta investigación tuvo como objetivo determinar que el uso de la canción es una estrategia lúdica que aporta a la enseñanza de los estudiantes de subnivel de preparatoria para adquirir un mejor aprendizaje. Para ello, se aplicó una investigación de carácter descriptiva. Los resultados obtenidos de esta indagación demostraron que la aplicación de la música mejora el proceso de aprendizaje de los estudiantes, por lo que se plantea una propuesta integrada por tres talleres. Seguidamente, se concluye que la música es un recurso acertado que mejora la actitud, motivación y las áreas cognitivas de los estudiantes.

Descriptores: Música; educación musical; aprendizaje activo. (Palabras tomadas del Tesauro UNESCO).

\begin{abstract}
The application of playful strategies to improve teaching processes in educational institutions is timely, because it transforms the transmission of knowledge into interesting processes. Therefore, this research aimed to determine that the use of song is a playful strategy that contributes to the teaching of high school sublevel students to acquire better learning. For this, a descriptive investigation was applied. The results obtained from this investigation showed that the application of music improves the learning process of students, which is why a proposal made up of three workshops is proposed. Next, it is concluded that music is a successful resource that improves the attitude, motivation and cognitive areas of students.
\end{abstract}

Descriptors: Music; music education; activity learning. (Words taken from the UNESCO Thesaurus). 


\section{INTRODUCCIÓN}

Los niños del mundo tienen derecho aprender y a educarse bajo parámetros preestablecidos, por lo que es responsabilidad de las autoridades educativas y de los maestros buscar métodos validados de enseñanza, que impulsen el desarrollo de habilidades y destrezas sustanciales en la vida académica y profesional (Fondo de las Naciones Unidas, UNICEF, 2019).

Por otro lado, (Salazar-Gutiérrez, 2016), en el ámbito educativo se ha detectado la necesidad de practicar estrategias metodológicas que ubiquen a los estudiantes en el centro del proceso de enseñanza, para que asuman su rol protagónico y sean sujetos activos en su aprendizaje, por lo que es necesario propiciar escenarios con condiciones integrales, que forjen múltiples destrezas en los aprendices, con el fin de formarlos con capacidad crítica para enfrentarse al mundo exterior forjando relaciones humanas eficientes.

Por otro lado, (Bermell et al. 2016), subrayan que los educadores generalmente están en la búsqueda constante de estrategias de enseñanza - aprendizaje que permitan distribuir y organizar de forma oportuna la información y el conocimiento escolar, que será trasmitido a los estudiantes de manera integral hasta que logren interiorizar conocimientos significativos. Así mismo, (Botella \& Marín, 2016), expresan que la música es una estrategia pedagógica valiosa, con gran independencia, fortalecida en su metodología por lo que la consideran muy interesante para forjar motivación e interés en los educandos.

Por lo tanto, (Magán-Hervas \& Gértrudix-Barrio, 2017), señalan que uno de los grandes desafíos de las instituciones educativas es practicar estrategias apropiadas de enseñanza que ofrezcan múltiples ventajas a los estudiantes, en este sentido, (NavarroSolís, 2017), alega que la música es un recurso valioso y novedoso que tiene impacto positivo en el desarrollo cognitivo, porque estimula el hemisferio izquierdo del cerebro y otras áreas que participan directamente en el discernimiento de información. 
En base a lo que mencionan, (Amaya \& Mardones, 2017), los docentes como principales transmisores de conocimientos y guías del proceso de enseñanza tienen la obligación de facilitar el aprendizaje a los estudiantes utilizando recursos o métodos pedagógicos, que contengan una metodología apropiada, para los diversos tipos de retentiva o asimilación de conocimientos, de esta forma los estudiantes lograrán construir procesos de aprendizaje significativos y representativos en la adquisición e interiorización de contenidos académicos.

En consideración, (Navarro-Solís, 2017), manifiesta que la música mejora los ambientes escolares, transformándolos en lugares atractivos y relajados, que fomentan en los alumnos el entusiasmo por permanecer en el aula, escuchando y aprendiendo lo que el educador transmita. Asimismo, las canciones encienden la chispa dinamizadora de todas las zonas que impulsan el desarrollo intelectual y de otras áreas motoras, sociales y de logro de lenguaje, lectura y escritura. En complemento, (Bermell et al. 2016), señalan que la música al constituirse en el material didáctico central ayuda a adquirir patrones de expresión, acentos y ritmos, que facilitarán la adquisición de vocabulario y competencias de lectura desde los primeros niveles de escolarización, también fomenta la concentración y creatividad e incluso ayuda a los estudiantes que padecen de trastornos de aprendizaje como la dislexia.

Ahora bien, es preciso señalar que en el subnivel de preparatoria en la Provincia de El Oro existe una pobre metodología de enseñanza, tanto así que los infantes pasan a otros años con varios vacíos en temáticas básicas, por lo que es necesario que los educadores empleen estrategias novedosas, que forjen un aprendizaje autoconsciente, potencializado y encaminado a convertirse en conocimientos que duren largos períodos en la memoria de los estudiantes. Además, los estudiantes al recibir las clases están desmotivados y desenfocados de la información que el educador está transmitiendo, por ello este artículo de investigación tiene como objetivo determinar que el uso de la canción es una estrategia lúdica que aporta a la enseñanza de los estudiantes de subnivel de preparatoria para adquirir un mejor aprendizaje, debido a que es favorable para generar 
ambientes cálidos y armónicos dentro del aula, porque por medio de las melodías se logran fortalecer los sentidos sensoriales y el área socio y afectiva de los infantes.

\section{Referencial teórico}

\section{Estrategias De Aprendizaje}

Las estrategias de aprendizaje que se utilizan en el ámbito educativo son procesos que se emplean para facilitarlo intencionalmente de donde los estudiantes extraen recursos para fortalecer sus enseñanzas, porque brindan una serie de tácticas que generan facilidades para reconocer e interiorizar información de forma oportuna, por ello su puesta en marcha es fundamental en el área académica. Considerando las palabras de (Roux \& Anzures, 2015), las estrategias de aprendizaje son procesos estructurados consientes e intencionales que los educadores utilizan para transmitir y recuperar información de manera coordinada con el propósito de alcanzar algún fin o demanda preestablecida que dependerá además de factores exógenos como las características del contexto educativo donde se está desarrollando el proceso de enseñanza.

Mientras que, (Visbal et al. 2017), expresan que las estrategias de enseñanza son operaciones mentales que se enmarcan en conductas y pensamientos que los educandos emplean cuando atraviesan por procesos de aprendizaje, para codificar la información o contenidos de acuerdo a la necesidad de cada uno con carácter intencional o propositivo, que los facultará adicionalmente para almacenar aprendizajes significativos. En base a estas definiciones las estrategias de enseñanza son procesos secuenciales y estructurales con planes dirigidos o preestablecidos para conseguir metas o propósitos educativos que son practicados de forma intencional y que se implican directamente en la toma de decisiones de los estudiantes al momento de interiorizar información validada o significativa para ellos, que generalmente lleva implícito un plan de acción. 


\section{La música}

De acuerdo con (Navarro-Solís, 2017), la música es considerada un lenguaje universal presente en todas las culturas a nivel mundial desde muchos atrás, tanto así que también se la define como un medio de expresión y comunicación. Según (Bermell et al. 2016), la canción está compuesta por bases matemáticas y aritméticas, por ello ayudan a desarrollar el pensamiento lógico - matemático en las personas que la practican. Desde el ámbito educativo la música es un recurso que estimula diversas facultades de los aprendices, como la imaginación, orden y la creatividad, porque en el despliegue de las melodías se estimulan diversos sentidos, que permite a sus zonas cognitivas funcionar de forma sistemática y encaminadas a tener mayor facilidad, para adquirir sus propios aprendizajes (UNESCO, 1980).

Tanto así, que (Arguedas-Quesada, 2015), aduce que la música al ser una locución artística posibilita su manipulación en el área pedagógica, porque beneficia la parte intelectual, motriz y de expresión en los educandos de edad subnivel de primaria, a través de la consolidación de métodos cognitivos, como la memoria, la atención, la percepción y la motivación. De hecho, el canto puede constituirse en el medio para el desarrollo de habilidades en los estudiantes, contribuyendo al crecimiento integral en cada una de las etapas de los niños y niñas.

Según (Botella et al. 2016), la música permite crear efectivos procesos cognitivos, creativos, científicos y morales en los educandos e incluso se ha señalado que el canto provoca que el universo de la razón, la enseñanza del lenguaje, las cifras, símbolos y el uso de la capacidad se desarrollen aceleradamente. Además, beneficia la memorización de citas y la vigorización de los mensajes e inclusive el aprendizaje de otros idiomas. En base a lo que menciona (Botella et al. 2016), la música tiene las siguientes características:

Parte de la Cultura: Cada pueblo o culturas humanas se acogen a manifestaciones musicales que expresan vivencias o historias a través de dimensiones rítmicas, melódicas y armónicas, que tienen diversas escalas musicales, pero que su propósito es 
el mismo. La música como parte integral de la cultura permite a los individuos de diferentes edades entender el entorno que los rodea, relacionarse y crear vínculos con la comunidad y entre las principales instituciones sociales que son la familia y la escuela. Forma de Comunicar: La música facilita el lenguaje interpersonal y transforma maneras de pensar, sentir o actuar a través de sus melodías que suelen ser transmisoras de letras que generan sensibilidad y llegan directamente al subconsciente de las personas, propiciando estados de análisis de diversas situaciones del mundo. Por lo tanto, es menester que en los centros educativos se creen espacios didácticos donde se desarrolle el lenguaje artístico a través de diversas actividades.

Al practicarse el lenguaje artístico en las aulas se desarrolla un proceso de enseñanza y aprendizaje cargado de estimulaciones sensitivas que se generan por las dinámicas del grupo, por ello es factible que la familia desde edades tempranas acceda a la música, generando experiencias previas valiosas, que servirán como base para futuros aprendizajes (Miñan-Aguacondo \& Espinoza-Freire, 2020).

Expresión Personal: La música favorece la expresión de emociones e ideas a través de la manipulación de sonidos y melodías, que fomentan experiencias significativas impulsadoras de la expresión de los seres humanos. Por ello, es primordial que en las instituciones educativas se promuevan actividades que generen habilidades expresivas como tocar instrumentos, bailar o cantar, que permiten fortalecer su desarrollo interpersonal y lingüístico.

Aumenta la Imaginación: Generalmente la imaginación y la creatividad en el contexto académico se relacionan directamente con la práctica de actividades artísticas, que son esenciales en la transformación educativa por la que se está atravesando en el mundo siendo la música una de las actividades principales que desarrolla en los estudiantes la imaginación, facultándolos para tomar decisiones y solucionar problemas inesperados en diversos ámbitos.

Desarrollo Humano: La música tiene características que se potencializan a través del desarrollo humano y que despliegan cualidades de los individuos, fomentando actitudes 
o competencias que aportan sustancialmente al fortalecimiento y realización personal como la capacidad de escuchar, concentración, perseverancia, autoestima e incluso disciplina, que se practica para crear ambientes caracterizados por la tolerancia. Para (Teruel \& Savón, 2018), la música tiene una metodología flexible de aplicación en el aula como las descritas en la Tabla 1.

\section{Tabla 1.}

Funciones de la música.

\begin{tabular}{cl}
\hline Función ambiental & $\begin{array}{l}\text { Se utiliza para crear un ambiente de trabajo agradable en el aula e } \\
\text { incluso al momento de que los estudiantes realicen alguna lectura } \\
\text { expresiva. }\end{array}$ \\
\hline Función formativa & $\begin{array}{l}\text { Se utiliza las canciones que transmiten alguna información, mensaje } \\
\text { o valores a los estudiantes. }\end{array}$ \\
\hline Función expresiva & $\begin{array}{l}\text { Se la utiliza en alguna actividad con el propósito de que exterioricen } \\
\text { sus sentimientos o emociones retraídas. }\end{array}$ \\
\hline Función reflexiva & $\begin{array}{l}\text { Se utiliza las canciones en lugares o espacios donde los estudiantes } \\
\text { puedan vivir experiencias nuevas. }\end{array}$ \\
\hline Función imaginaria & $\begin{array}{l}\text { Se utiliza las canciones para que los estudiantes tengan momentos } \\
\text { de transición, desprendiéndose del mundo real por un momento, } \\
\text { creando imágenes y escenarios llenos de fantasías, que ayuden a } \\
\text { relajarse y aperturar espacios en su memoria. }\end{array}$ \\
\hline Función de & $\begin{array}{l}\text { Se utilizan las canciones para dar secuencia a diversos tiempos de la } \\
\text { clase, tanto así que al inicio o al cierre se pueden utilizar melodías } \\
\text { que reflejen esos momentos. }\end{array}$ \\
\hline encadenamiento & $\begin{array}{l}\text { Los estudiantes cuando escuchan una determinada pieza musical lo } \\
\text { asocian a determinados contenidos que se interiorizan de forma } \\
\text { sintética con su universo racional y emocional. }\end{array}$ \\
\hline Función memorística
\end{tabular}

Fuente: Adaptado de Teruel et al. (2018). 
La práctica de la música en el proceso de enseñanza da continuidad a otras herramientas utilizadas, para transmitir diversos contenidos, como lo indica la Tabla 2.

Tabla 2.

Tipología de la música.

$\begin{array}{ll}\text { Tiempo } & \begin{array}{l}\text { Que llueva que llueva, Son } 7 \text { días, Sol solito, buenas } \\ \text { noches. }\end{array}\end{array}$

Animales Vaca lechera, el señor don gato, La rana sentada.

Números, tono y colores Un elefante, Las vocales, los globos son.

Lenguaje Aserrín-aceran, Debajo un botón ton-ton, Muñeca azul.

Fuente: Adaptado de Teruel et al., 2018.

\section{Efecto Mozart}

En base a lo que menciona, (Oliven, 2015), durante mucho tiempo se ha venido aduciendo que la música propicia ambientes de entendimiento, despeja la mente y refuerza el sistema cognitivo de los individuos, aún más cuando es escuchada y practicada desde los primeros años de vida, sustentándose en esta aseveración nace una teoría denominada el Efecto Mozart que promulga las ventajas de que los bebes escuchen música clásica.

Las melodías clásicas de Wolfgang Amadeus Mozart (1576-1791) tienen una repercusión positiva en la mente los niños, jóvenes o adultos e incluso si es escuchada desde el vientre de la madre o en contextos que necesitan concentración o capacidad de análisis, porque dinamiza zonas cognitivas que impulsan la retentiva o almacenamiento de información de forma eficiente y duradera (Oliven, 2015). 


\section{METODOLOGÍA}

Esta investigación fue de carácter descriptiva no experimental, se empleó una población de 44 estudiantes del primer año de Educación Básica de dos paralelos de una institución educativa de la Provincia de El Oro - Santa Rosa, pero a través de un muestreo no probabilístico el investigador seleccionó 14 estudiantes, 7 de género femenino y 7 de género masculino basado en su juicio subjetivo.

El instrumento fue un cuestionario de 15 preguntas en escala Likert con los siguientes niveles de respuesta Siempre (1), Frecuentemente (2), Ocasionalmente (3) y Nunca (4) que sirvieron para determinar la forma en que los niños reaccionaban a las clases cuando el educador utilizaba la música infantil para trasmitir o enseñar algún contenido.

Este instrumento de investigación fue validado a través del cálculo de fiabilidad de Alfa de Cronbach con un resultado de 0,787 mientras que las quince variables en el test Shapiro Wilk fueron paramétricas porque tienen un nivel de significancia menores de 0,05 . Finalmente, se aplicó un análisis estadístico inferencial a través el programa SPSS. Se procesó la información en base a estadística descriptiva.

\section{RESULTADOS}

Los resultados cualitativos fueron obtenidos con la aplicación de la ficha de observación a 14 estudiantes de subnivel de primaria de una institución educativa de la Provincia de El Oro - Santa Rosa, los datos recabados fueron ingresados y procesados en el software SPSS con la finalidad de analizar de qué manera la aplicación de la música como estrategia metodológica fortalece el desarrollo integral en los estudiantes.

Para ello, se aplicó a las quince variables el test Shapiro Wilk que arrojó como resultado que son paramétricas porque tienen un nivel de significancia menor de 0,05 y para probar la hipótesis se aplicó la prueba $T$ con un $p$-valor de $a=<0.005$ para que la hipótesis sea afirmativa y que sea nula con $p$-valor $=>0.05$. 
Revista Arbitrada Interdisciplinaria KOINONIA

Año VI. Vol VI. N4. Edición Especial: Educación III. 2021

Hecho el depósito de Ley: FA2016000010 ISSN: 2542-3088

FUNDACIÓN KOINONIA (F.K). Santa Ana de Coro. Venezuela.

Tabla 3.

Resultados estadísticos prueba T.

\begin{tabular}{ccccccccc}
\hline Preguntas & \multicolumn{2}{c}{ recdid_mus } & \multicolumn{2}{c}{ mot_mus } & \multicolumn{2}{c}{ habcog_mus } & \multicolumn{2}{c}{ ingles_musica } \\
\hline Test & Pre & Post & Pre & Post & Pre & Post & Pre & Post \\
\hline Número & 14 & 14 & 14 & 14 & 14 & 14 & 14 & 14 \\
\hline Media & 1,29 & 2,00 & 1,29 & 1,93 & 1,21 & 2,07 & 1,50 & 2,29 \\
\hline $\begin{array}{c}\text { Sig. } \\
\text { (bilateral) }\end{array}$ & 0,003 & 0,003 & 0,005 & 0,005 & 0,000 & 0,000 & 0,003 & 0,003 \\
\hline $\begin{array}{c}\text { Desviación } \\
\text { estándar }\end{array}$ & 0,611 & 0,555 & 0,611 & 0,555 & 0,579 & 0,475 & 0,650 & 0,611 \\
\hline $\begin{array}{c}\text { Error de la } \\
\text { media }\end{array}$ & 0,163 & 0,148 & 0,163 & 0,127 & 0,155 & 0,127 & 0,174 & 0,163 \\
\hline
\end{tabular}

Fuente: Estadísticas.

\section{Leyenda}

recdid_mus: Demuestran buena actitud por la música al utilizarla como recurso didáctico mot_mus: Participa de forma activa en las actividades de la clase motivados por la música infantil habcog_mus: Mediante el uso de la música infantil los niños demuestran mejores habilidades cognitivas ingles_musica: Mediante la utilización de la música infantil los estudiantes adquieren aprendizajes de inglés

Los resultados expuestos en la Tabla 3 evidencian que los estudiantes demuestran buena actitud al utilizar la música como recurso didáctico, por ello en el pretest la media es de 1,29 y en el post test es de 2,00 demostrando que al utilizar la música los infantes tienen mejor predisposición, que al mismo tiempo se enmarca en el índice obtenido en la prueba $T(0,003)$ que señala una hipótesis afirmativa.

En la interrogante participa de forma activa en la clase motivados por la música infantil en el pretest la media fue de 1,29 y en el post test de 1,93 denotando que los aprendices incrementan su motivación cuando aprenden es espacios con melodías musicales, que se respalda en la prueba $T(0,005)$ aceptando la hipótesis. 
Asimismo, en la pregunta referente a que mediante la música infantil los niños demuestran mejores habilidades cognitivas la media en el pretest fue de 1,21 y en el post test de 2,07 demostrando que la música estimula zonas cerebrales de los individuos, avalándose en la prueba T $(0,000)$ de aceptación de la hipótesis.

En cambio, en la interrogante mediante la utilización de la música infantil los estudiantes adquieren aprendizajes de inglés en el pretest fue de 1,50 y en el post test de 2,29, denotando que los estudiantes adquieren fácilmente nuevos aprendizajes, aunque sean complejos con la utilización de melodías, respaldándose en la prueba $T(0,003)$ que acepta la hipótesis.

\section{PROPUESTA}

En base a la investigación planteada se diseñó y planteó una propuesta de intervención basada en la música infantil como instrumento de mejora de los procesos de aprendizaje en el Subnivel de Preparatoria, porque ayuda a trabajar diversas áreas como aspectos comunicativos, adquisición de vocabulario, desarrollo cognitivo, memorización e incluso el despliegue de habilidades de lectoescritura, por lo que se convierte en un recurso didáctico valioso que aprovecha al máximo cada una de las capacidades de los estudiantes. Por lo tanto, la siguiente propuesta está compuesta por talleres que utilizan a la música infantil para motivar y entretener a los estudiantes en las clases, así como también para fortalecer aspectos de lenguaje y cognitivos. Para ello se ha diseñado la propuesta de intervención detallada en la Tabla 4. 
Revista Arbitrada Interdisciplinaria KOINONIA

Año VI. Vol VI. N4. Edición Especial: Educación III. 2021

Hecho el depósito de Ley: FA2016000010 ISSN: 2542-3088

FUNDACIÓN KOINONIA (F.K). Santa Ana de Coro. Venezuela.

Jener Fabricio Chica-Correa; María Isabel Álvarez-Lozano; Claudio Fernando Guevara-Vizcaíno

Tabla 4.

Propuesta de intervención.

\begin{tabular}{|c|c|c|c|c|}
\hline \multicolumn{5}{|c|}{ Taller 1. Pronunciación } \\
\hline Ámbitos & Destrezas & $\begin{array}{l}\text { Recursos } \\
\text { materiales }\end{array}$ & Actividad & $\begin{array}{l}\text { Criterios de } \\
\text { evaluación }\end{array}$ \\
\hline $\begin{array}{l}\text { Comprensión y } \\
\text { expresión del } \\
\text { lenguaje } \\
\text { Expresión } \\
\text { artística }\end{array}$ & $\begin{array}{l}\text { Expresarse } \\
\text { correctamente } \\
\text { a través de una } \\
\text { adecuada } \\
\text { pronunciación. } \\
\text {-Desarrollar } \\
\text { movimientos } \\
\text { corporales. }\end{array}$ & $\begin{array}{l}\text {-Laminas. } \\
\text {-Canción. } \\
\text {-Grabadora. }\end{array}$ & $\begin{array}{l}\text { Interpretación de la } \\
\text { canción el "loro } \\
\text { Lorenzo" que sirve para } \\
\text { mejorar la pronunciación } \\
\text { de la letra "r" y mejora la } \\
\text { retentiva y creatividad. } \\
\text { Letra } \\
\text { Al loro Lorenzo } \\
\text { Le gusta la luna } \\
\text { Al tope de la loma } \\
\text { La mira en la laguna } \\
\text {...... }\end{array}$ & $\begin{array}{l}\text {-Se expresa } \\
\text { adecuadamente. } \\
\text {-Tiene movimientos } \\
\text { corporales de } \\
\text { acuerdo al ritmo. } \\
\text { Técnica: } \\
\text { Observación }\end{array}$ \\
\hline
\end{tabular}

Taller 2. Recociendo la naturaleza

\begin{tabular}{|c|c|c|c|c|}
\hline Medio natural & $\begin{array}{l}\text { Reconocer los } \\
\text { elementos de la } \\
\text { naturaleza. }\end{array}$ & $\begin{array}{l}\text {-Canción. } \\
\text {-Grabadora. }\end{array}$ & $\begin{array}{l}\text { Interpretación de la } \\
\text { canción "el ferrocarril" } \\
\text { que ayuda a mejorar los } \\
\text { problemas de } \\
\text { articulación y omisión de } \\
\text { fonemas y fomenta la } \\
\text { creatividad. } \\
\text { Letra } \\
\text { Chucu chucu } \\
\text { El ferrocarril, por los } \\
\text { rieles recorre } \\
\text { Mirando el } \\
\text { atardecer...... }\end{array}$ & $\begin{array}{l}\text {-Reconoce } \\
\text { adecuadamente los } \\
\text { elementos de la } \\
\text { naturaleza. } \\
\text { Técnica: } \\
\text { Observación }\end{array}$ \\
\hline \multicolumn{5}{|c|}{ Taller 3. Regulando mi estado de ánimo } \\
\hline $\begin{array}{l}\text { Identidad y } \\
\text { autonomía. }\end{array}$ & $\begin{array}{l}\text { Lograr } \\
\text { manifestar } \\
\text { libremente las } \\
\text { emociones y } \\
\text { expresiones } \\
\text { orales o } \\
\text { gestuales. }\end{array}$ & $\begin{array}{l}\text {-Canción. } \\
\text {-Grabadora. }\end{array}$ & $\begin{array}{l}\text { Interpretar la canción } \\
\text { "Todas las mañanas" } \\
\text { que fomenta la identidad } \\
\text { y autonomía de los } \\
\text { estudiantes. } \\
\text { Letra } \\
\text { Todas las mañanas }\end{array}$ & $\begin{array}{l}\text {-Expresa libremente } \\
\text { sus emociones. } \\
\text { Técnica: } \\
\text { Observación }\end{array}$ \\
\hline
\end{tabular}


Elaboración: Los autores.

Las actividades detalladas en la Tabla 4 se planificaron con tres momentos en la clase que son:

Inicio: Para iniciar la clase el educador saludó a los estudiantes utilizando melodías musicales para ambientarlos con el recurso didáctico que se iba a utilizar.

Desarrollo: En este lapso de tiempo se aplicó cada taller descrito en la Tabla 4 con los ámbitos, destrezas y recursos detallados durante 25 minutos.

Cierre: Para culminar se plantearon unos criterios de evaluación estipulados en la Tabla 4 a través de una ficha de observación que fue aplicada a los estudiantes al culminar los talleres.

\section{CONCLUSIONES}

Las instituciones educativas actualmente están llamadas a utilizar estrategias vanguardistas, que permitan desarrollar un proceso de enseñanza-aprendizaje apropiado con múltiples beneficios para los educandos, porque la implementación de metodologías integrales, estimulará el sistema cognitivo y otras áreas muy necesarias durante el proceso de escolarización, además tienen la capacidad de captar su atención y motivarlos para seguir adquiriendo conocimientos.

La música es considerada un lenguaje universal que expresa en sus letras cultura y sedimenta conocimientos, por ende, se convierte en un canal efectivo que fomenta la asimilación de contenidos de forma rápida, por ello su utilización en los procesos de enseñanza-aprendizaje es favorecedor, debido a que impulsa la creatividad, potencia la agilidad cerebral y ayuda al momento de querer resolver problemas matemáticos por sus funciones de relajación. 
Es preciso señalar que al aplicar una ficha observación a 14 estudiantes de Subnivel de Preparatoria durante una clase donde se aplicó la música como recurso didáctico se ha evidenciado que tienen una mayor predisposición para aprender, buena actitud, participación activa y desarrollo de habilidades cognitivas, además aprenden el idioma inglés con facilidad, denotando que la aplicación de esta metodología de enseñanza trae beneficios a los estudiantes.

Por ello, en base a los resultados obtenidos se ha realizado una propuesta de intervención focalizada en utilizar la música infantil como recurso sustancial, para mejorar los procesos de aprendizaje del Subnivel de Preparatoria, debido a que las sesiones se diseñaron para mejorar el área lingüística y cognitiva de los educandos, como la adquisición de vocabulario, comunicación, memoria e incluso fomenta habilidades para leer y escribir.

Por ello, se establece que la implementación de la música como estrategia metodológica para fortalecer el desarrollo integral en los estudiantes del Subnivel de Preparatoria es un recurso acertado, porque los estudiantes mejoran su actitud, motivación, áreas cognitivas y tienen predisposición para adquirir nuevos aprendizajes, aunque sean complejos. Por ello, es sustancial que los educadores estén abiertos a nuevas posibilidades de rediseñar sus formas de enseñar, porque la forma tradicional no tiene efectos positivos en la comunidad estudiantil, caso contrario sucede cuando se aplican recursos o métodos vanguardistas que captan su atención y los convierte en los protagonistas de sus propios aprendizajes.

\section{FINANCIAMIENTO}

No monetario.

\section{AGRADECIMIENTO}

A la Universidad Católica de Cuenca; por todo el apoyo brindado en la motivación y desarrollo de esta investigación. 
Revista Arbitrada Interdisciplinaria KOINONIA

Año VI. Vol VI. N4. Edición Especial: Educación III. 2021

Hecho el depósito de Ley: FA2016000010

ISSN: 2542-3088

FUNDACIÓN KOINONIA (F.K). Santa Ana de Coro. Venezuela.

Jener Fabricio Chica-Correa; María Isabel Álvarez-Lozano; Claudio Fernando Guevara-Vizcaíno

\section{REFERENCIAS CONSULTADAS}

Amaya, M. \& Mardones, M. (2017). La música como estrategia didáctica para la enseñanza de una segunda lengua, una revisión teórica. Un acercamiento al concepto de didáctica [Music as a didactic strategy for teaching a second language, a theoretical review. An approach to the concept of didactics]. Foro Educacional, 20(0717-2710), 55-76. https://n9.cl/klegp

Arguedas-Quesada, C. (2015). Educación musical, desarrollo infantil y adolescente y enfoque de derechos humanos: Una reseña bibliográfica [Music education, child and adolescent development and a human rights approach: A bibliographic review]. Revista Educación, 39(2), 79 - 86. https://doi.org/10.15517/revedu.v39i2.19899

Fondo de las Naciones Unidas, UNICEF. (2019). Informe anual 2019 [Annual report 2019]. Recuperado de https://n9.cl/4gu0m

Bermell, Á., Brull, V. A. y Bernabé Villodre, M. (2016). Impacto de la educación musical para la competencia social en educación infantil [Impact of music education for social competence in early childhood education]. Opcion, 32(81), 104 - 128. https://n9.cl/f89fz

Botella, A. M. \& Marín, P. (2016). La utilización del musicomovigrama como recurso didáctico para el trabajo de la audición atenta, comprensiva y activa en educación primaria [The use of the musicomovigram as a didactic resource for the work of attentive, comprehensive and active listening in primary education]. Cuadernos de Música, Artes Visuales y Artes Escénicas, 11(2), 215 - 237.

Magán-Hervás, A. y Gértrudix-Barrio, F. (2017). Influencia de las actividades audiomusicales en la adquisición de la lectoescritura en niños y niñas de cinco años [Influence of audio-musical activities on the acquisition of literacy in five-year-old boys and girls]. Revista Electrónica Educare, 21(1), 12 - 23. https://doi.org/10.15359/ree.21-1.15

Miñan-Aguacondo D. \& Espinoza-Freire, E. (2020). La pedagogía musical [Music pedagogy]. Revista Universidad y Sociedad, 12(5), 454-460.

Navarro-Solís, J. L. (2017). Pautas para la aplicación de métodos de enseñanza musical desde un enfoque constructivista [Guidelines for the application of music teaching methods from a constructivist approach]. Revista Electrónica de Investigación Educativa, 19(3), 143 - 157. https://doi.org/10.24320/redie.2017.19.3.675 
Oliven, R. G. (2015). The imaginary of Brazilian popular music [Lo imaginario de la música popular brasileña]. Vibrant: Virtual Brazilian Anthropology, 8(1), 170-207. https://doi.org/10.1590/s1809-43412011000100007

Roux, R. \& Anzures, E. E. (2015). Estrategias de aprendizaje y su relación con el rendimiento académico en estudiantes de una escuela privada de educación media superior [Learning strategies and their relationship with academic performance in students of a private upper secondary school]. Revista Electrónica "Actualidades Investigativas En Educación," 15(1), 1-16. https://n9.cl/to4gp

Salazar-Gutiérrez, N. P. (2016). Músicas tradicionales en espacios académicos: la rueda de gaita como experiencia de aprendizaje [Traditional music in academic spaces: the bagpipe wheel as a learning experience]. Civilizar, 16(31), 2 - 18. https://doi.org/10.22518/16578953.650

Teruel, E. \& Savón, C. (2018). Una metodología para el tratamiento a los medios expresivos de la música los medios expresivos de la música [A methodology for treating the expressive means of music the expressive means of music]. EduSol, 18(64), 12 -34. https://n9.cl/p180o

UNESCO. (1980). The child and play Theoretical approaches and teaching applications. Recuperado de https://n9.cl/pbcri

Visbal, D., Mendoza, A. \& Santana, S. (2017). Estrategias de aprendizaje en la educación superior [Learning strategies in higher education]. Sophia, 13(2), 70-81. https://n9.cl/3gfzt 